\title{
Richard Teschner (1879-1948) et le théâtre de marionnettes artistique entre Prague et Vienne
}

\section{Céline Trautmann-Waller}

\section{(2) OpenEdition}

1 Journals

Édition électronique

URL : https://journals.openedition.org/austriaca/936

DOI : 10.4000/austriaca.936

ISSN : 2729-0603

Éditeur

Presses universitaires de Rouen et du Havre

\section{Édition imprimée}

Date de publication : 1 décembre 2019

Pagination : 105-116

ISBN : 979-10-240-1454-8

ISSN : 0396-4590

\section{Référence électronique}

Céline Trautmann-Waller, «Richard Teschner (1879-1948) et le théâtre de marionnettes artistique entre Prague et Vienne », Austriaca [En ligne], 88-89 | 2019, mis en ligne le 31 décembre 2020, consulté le 24 octobre 2022. URL : http://journals.openedition.org/austriaca/936 ; DOI : https://doi.org/ 10.4000/austriaca.936 


\section{Richard Teschner (1879-1948) et le théâtre de marionnettes artistique entre Prague et Vienne}

Richard Teschner est connu dans le domaine du théâtre de marionnettes pour avoir élevé celui-ci au niveau d'un art total. Fabriquant lui-même ses marionnettes, leurs costumes, les scènes, les décors, les accessoires et jusqu'aux instruments de musique sur lesquels il accompagnait les représentations, il devient, notamment avec les scènes Der Goldene Schrein et Der Figurenspiegel à Vienne, le représentant sans doute le plus connu du théâtre de marionnettes artistique, un genre souvent associé à l'espace centre- et est-européen.

Étudiant des Beaux-Arts à Prague, Teschner ne se destinait pourtant pas au départ au théâtre de marionnettes, mais développa une production prolifique dans différents domaines, allant de la peinture au graphisme et aux arts décoratifs, en s'efforçant de se créer une identité artistique dans le milieu des Allemands de Bohême, de leurs associations, de leurs revues et de leurs lieux d'exposition. C'est tout d'abord ce milieu, les tensions entre différentes générations d'Allemands de Bohême et entre celles-ci et les défenseurs d'un réveil national tchèque, ainsi que ses répercussions sur la vie artistique, qu'il faut retracer pour comprendre la jeunesse de Teschner. L'installation à Vienne en 1909, qui, au-delà du cas individuel de Teschner, est caractéristique de toute une génération, peut se comprendre comme la recherche d'une issue hors de ces conflits. À ses débuts à Vienne, Teschner n'en continuera pas moins d'être vu dans les milieux artistiques de la capitale autrichienne avant tout comme un "Allemand de Bohême». L'hypothèse de cette contribution est que c'est sur cet arrière-plan que prend sens la «révélation» que connut Teschner au contact du théâtre de marionnettes indonésien, qui lui permit de "recycler» et de moderniser un héritage tchèque, teinté de patriotisme local, au contact des milieux artistiques viennois attirés par les arts orientaux. Autant le statut complexe des marionnettes de Teschner entre art, arts populaires et arts appliqués, que leur style très particulier, mêlant le fantastique praguois 
à un exotisme asiatique revu et corrigé, s'inscrivent dans une tension entre Prague et Vienne. Le cas de Teschner pose ainsi à sa manière la question des relations et des tensions entre les deux villes, des configurations spécifiques du rapport entre avant-garde et folklore qui s'y dessinent, ainsi que du rôle que joua l'arrière-plan culturel de chacune d'entre elles dans l'appropriation des traditions asiatiques du théâtre de marionnettes par Teschner, vue parfois comme l'émergence d'un théâtre de marionnettes globalisé.

\section{Richard Teschner et les dilemmes des jeunes artistes germanophones en Bohême}

La jeunesse de Teschner a pu être caractérisée comme une période marquée par une série de «ruptures ${ }^{1} »$. Né en 1879 à Karlsbad/Karlovy Vary, installé ensuite à Leitmeritz/Litoměřice, où son père ouvre un atelier de lithographie, il étudie à l'Académie des Beaux-Arts de Prague entre 1896 et 1899 avant de partir pour Vienne avec le projet d'y poursuivre ses études également à l'Académie des Beaux-Arts. Ce projet échoue et Teschner se replie sur l'École des arts appliqués, où il étudie chez Karl Karger de 1900 à 1901, vivant cette situation plus ou moins comme un échec $^{2}$. Ceci expliquerait son retour d'abord à Leitmeritz/Litoměrice, où il travaille dans l'atelier de son père, puis à Prague en 1902, grâce à une subvention de la Société pour la promotion de l'art, de la science et de la politique allemandes (Gesellschaft zur Förderung deutscher Kunst, Wissenschaft und Politik). Dès son retour à Prague, Teschner participe à la scène artistique germanophone praguoise autour des groupements Concordia, l'Association des artistes plasticiens allemands de Bohême (Verein deutscher bildender Künstler in Böhmen) et Jung Prag, n'excluant aucune de ces options en dépit des différences de génération et de tendances politiques qui caractérisent ces différents cercles. Avec le temps, il se rapproche toutefois nettement du groupe néo-romantique Jung Prag né vers 1898 autour d'Oskar Wiener et dont l'un des buts affichés était

1. Concernant la jeunesse de Teschner, voir Kurt Ifkovits, «Richard Teschners Prager Jahre», dans Kurt Ifkovits (éd.), „Mit diesen meinen zwei Händen..." Die Bühne des Richard Teschner, Wien, Österreichisches Theatermuseum, 2013, p. 17-41.

2. Dans une lettre datée approximativement du 16 octobre 1900, il raconte à son ami Karl Wilfert son arrivée à Vienne et ses démarches auprès de toute une série de professeurs de l'Académie des Beaux-Arts puis de la Kunstgewerbeschule. Voir «Richard Teschner an Karl Wilfertd. J., undat. (wohl 16. Oktober 1900)», dans Kurt Ifkovits, „Mit diesen meinen zwei Händen...", op. cit., p. 14-15. 
de dépasser ce que la jeune génération analysait comme un provincialisme praguois, en ouvrant la vie artistique locale aux influences internationales. Teschner paraît s'épanouir dans ce cercle, caractérisé par des échanges importants entre littérature et arts plastiques : il illustre les productions littéraires de ses amis, crée des cartons d'invitation et des affiches pour leurs manifestations artistiques ${ }^{3}$.

Prague est marquée en cette fin de $\mathrm{XIX}^{\mathrm{e}}$ siècle par l'essor du nationalisme tchèque et les tensions avec la minorité allemande qui s'ensuivent. Celles-ci ont conduit notamment à la séparation del'université de Prague en une université germanophone et une université tchèque en 1882. En 1883, l'inauguration du Théâtre national est vécue comme le début d'une nouvelle ère. Les Allemands de Bohême cherchent, en réaction, à affirmer leur identité "germanique» et Teschner se voit régulièrement fêté dans ce contexte comme un "artiste allemand de Bohême». Sa proximité avec le monde des contes notamment est conçue comme typiquement germanique ${ }^{4}$. C'est ce que révèle également l'écho que rencontrent ses œuvres lors de différentes expositions. L'exposition germano-bohémienne (Deutschböhmische Austellung) à Reichenberg/Liberec en 1906, où il fête ses premiers grands succès, constitue de ce point de vue un cas particulièrement parlant. Comme il a été souligné, la ville de Reichenberg/Liberec s'efforce alors, depuis un certain moment déjà, de devenir la «métropole des Allemands de Bohême ${ }^{5}$ » et l'exposition de 1906 constitue sans doute l'une des illustrations les plus visibles de ce projet. La proposition en avait été faite par l'association pour le commerce (Gewerbeverein) de Reichenberg/Liberec dès 1902 et «[1]'exposition était censée devenir un étalage imposant de différents domaines du "travail allemand» issus de toute la Bohême» (Es sollte eine imposante Schau verschiedener Bereiche "deutscher Arbeit" aus ganz Böhmen werden) $)^{6}$. Comme l'expliquait le critique et journaliste Franz Servaes dans son compte rendu de l'exposition, les Allemands de Bohême étaient sensibles à la place que l'art jouait dans la conscience nationale tchèque et cher-

3. Ibid., p. 19.

4. Ibid., p. 21.

5. Tomáš Okurka, «Die Deutschböhmische Ausstellung in Reichenberg 1906», dans Kristina Kaiserová et Miroslav Kunštát (éds.), Die Suche nach dem Zentrum. Wissenschaftliche Institute und Bildungseinrichtungen der Deutschen in Böhmen (1800-1945), MünsterNew York, Schriftenreihe der Kommission für deutsche und osteuropäische Volkskunde in der Deutschen Gesellschaft für Volkskunde e.V., Bd. 96, 2014, p. 371-391, ici p. 371.

6. Ibid. 
chaient eux aussi à mettre l'art au service de leur identité complexe ${ }^{7}$. C'est également en 1906 que Teschner fonde avec son ami du groupe Jung Prag, le romancier Paul Leppin, la revue Wir qui a pu être définie comme "dernière tentative d'auto-affirmation" (letzter Selbstbehauptungsversuch) des Allemands de Bohême ${ }^{8}$. Teschner assume la responsabilité éditoriale de la partie artistique de la revue, dont seuls deux numéros parurent.

Quelle place revient au théâtre de marionnettes dans ce contexte? Selon différentes sources, Teschner aurait réalisé son premier théâtre de marionnettes dès 1904, peut-être plus par jeu qu'en songeant à en faire une occupation majeure9. Dès 1905, il aurait nourri néanmoins avec Gustav Meyrink, qui l'immortalisa sous les traits d'Athanasius Pernath dans son Golem (1914), le projet de créer un théâtre de marionnettes en collaboration avec la Wiener Werkstätte. C'est à cette époque que ses créations autour des marionnettes commencent à acquérir un véritable statut artistique. Dès 1906, il expose quelques marionnettes lors de l'exposition de Reichenberg/Liberec évoquée plus haut. Elles suscitent l'intérêt d'autres marionnettistes, notamment de Paul Brann qui tentait également à cette époque, d’une manière un peu différente, de développer un théâtre de marionnettes artistique. Tous deux partagent en tout cas l'idée que la marionnette est la seule réponse possible à la crise du théâtre ${ }^{10}$. Dans une lettre à Brann, Teschner développe en 1906 ses idées et ses projets concernant le théâtre de marionnettes : il s'oppose aux tendances naturalistes du théâtre de son époque et juge que tout est à recréer. Sa relation à la tradition des marionnettistes tchèques y apparait comme ambivalente. D’un côté Teschner juge que «dans le théâtre de marionnettes tel qu'il est présenté aujourd'hui sur les foires et dans les villages de Bohême par des personnes primitives, rien n'est bon que le principe». Plus loin, vers la fin de la lettre, il considère néanmoins, lors-

7. Franz Servaes, «Die Kunst auf der Reichenberger Ausstellung», Deutsche Arbeit. Monatsschrift für das geistige Leben der deutschen in Böhmen, vol. 11, n ${ }^{\circ}$ 5, 1906, p. 365-372, ici p. 365.

8. Kurt Krolop, «Zur Geschichte und Vorgeschichte der Prager deutschen Literatur des «expressionistischen Jahrzehnts», dans Eduard Goldstücker (éd.), Weltfreunde. Konferenz über die Prager Deutsche Literatur, Prague, Academia, Verlag der Tschechoslowakischen Akademie der Wissenschaften, 1967, p. 47-96, ici p. 53.

9. «Teschner (Richard)», dans Henryk Jurkowski et Thieri Foulc, Encyclopédie mondiale des arts de la marionnette, Montpellier, l'Entretemps, 2009, p. 695-696.

10. Mascha Erbelding, "„Alles wäre Kunst“. Über die Begegnung der Pioniere eines künstlerischen Puppentheaters Paul Brann und Richard Teschner», dans Kurt Ifkovits, „Mit diesen meinen zwei Händen...", op. cit., p. 67-69. 
qu'il explique comment il est parvenu à déterminer une taille optimale pour ses marionnettes : "J'ai effectué des études approfondies à ce sujet dans plusieurs théâtres de marionnettes existant à Prague. Prague était de manière générale un excellent terrain pour des études préparatoires aux marionnettes ${ }^{11}$.»

Teschner continue à exposer des marionnettes : à la deuxième Kunstausstellung deutsch-böhmischer Künstler à Karlsbad/Karlovy Vary en 1906, à la Kunstausstellung du Verein der deutschen bildenden Künstler in Prag en 1907 et lors de la Wiener Kunstschau en 1908, où il montre de nouveau, à côté de réalisations graphiques et de tableaux allégoriques en mosaïque de verres, des marionnettes, cette fois-ci les personnages de la pièce Le Fou et la Mort (1894) de Hofmannsthal. Edward Gordon Craig, grand collectionneur et connaisseur de marionnettes anglais, s'intéresse aux réalisations de Teschner dès 1905 et, selon certaines sources, il aurait puisé dans celles-ci l'idée de sa "surmarionnette" (Über-Marionette), une figure inanimée supérieure à la taille humaine et qui devait remplacer l'acteur dans le théâtre de l'avenir que Craig appelait de ses voeux. Dans les carnets de croquis de Craig concernant la «surmarionnette», certains ont cru reconnaître les marionnettes imaginées par Teschner pour Le Fou et la Mort ${ }^{12}$.

Le point d'orgue de cette période praguoise est sans doute néanmoins constitué par la réalisation en 1908 des décors pour la première présentation à Prague, au Théâtre allemand, de l'opéra de Debussy Pelléas et Melisande. Après celle-ci et de vains efforts pour obtenir un poste de professeur à l'Académie des Beaux-Arts et pour créer, avec Karl Wilfert, une académie d'art germanophone, Teschner part pour Vienne en 1909.

\section{La découverte des marionnettes asiatiques comme échappatoire au sort de l'«Allemand de Bohême» à Vienne}

On peut lire parfois que c'est grâce au soutien de Gustav Meyrink que Teschner serait entré en contact vers 1907-1908 avec la Wiener Werkstätte pour la réalisation d'un théâtre de marionnettes. En réalité ces contacts datent de 1905, année où Teschner réalise des gravures pour la Wiener

11. «Richard Teschner an Paul Brann, undat. (zwischen Mai und Juli 1906)», ibid., p. 62-65, ici p. 65.

12. Voir Patrick Le Boeuf, "On the Nature of Edward Gordon Craig's Über-Marionette», New Theatre Quarterly, vol. 26, n ${ }^{\circ}$, mai 2010, p. 102-114. 
$W_{\text {Werkstätte }}^{13}$. Après son installation à Vienne en 1909, Teschner, loin de se concentrer sur les marionnettes, développe toute une série d'activités. Il réalise des décors, des figurines et des réalisations graphiques, entre autres avec le membre de la Wiener Werkstätte Otto Prutscher. Il continue à être présenté dans les articles critiques avant tout comme un "Allemand de Bohême». On ne s'étonnera pas que ce soit le cas dans un article de 1911 consacré à Teschner et à ses activités à Vienne dans la revue d'art décoratif Deutsche Kunst und Dekoration ${ }^{14}$, puisque pour l'auteur, Franz Servaes, qui louait déjà Teschner lors de l'exposition de Reichenberg/Liberec, il s'agit sans doute de faire de la carrière artistique viennoise de ce dernier un outil de promotion de la culture allemande de/en Bohême. Le ton est différent dans un compte rendu de l'aménagement d'une chambre de dame dans la revue Dekorative Kunst, où Teschner est également présenté comme un Deutschböhme ${ }^{15}$. Car Hugo Haberfeld ajoute avec une pointe de rejet : «de même que Teschner en tant qu'homme n'a rien de "viennois", de même cet intérieur n'a pas le nouveau ton viennois» (sowie Teschner als Mensch nichts "Wienerisches hat" so auch dieses Interieur nicht die neue Wiener Note ${ }^{16}$ ).

Son mariage avec Emma Bacher-Paulick en 1911 resserre les liens de Teschner avec le cercle d'artistes autour de Gustav Klimt, liens attestés notamment par les séjours en commun près de l'Attersee durant la période estivale. Ceci explique aussi que dans certains articles Teschner ait pu être comparé à Klimt et décrit comme l'exact contraire de celui-ci : discipliné, introverti et pris dans des conventions anciennes, là où Klimt débordait de joie de vivre, se montrait sociable, chaotique et génial ${ }^{17}$. Cela n'empêcha pas les décors de Teschner et certaines de ses figures féminines de rappeler par certains aspects l'œuvre de Klimt. Dans les critiques concernant ses réalisations de l'époque, Teschner est généralement loué pour ses aptitudes techniques et artisanales. Ainsi

13. Gerd Pichler, «Richard Teschner und die Wiener Werkstätte. Der Einzug des Grotesken ins Kunstgewerbe der Wiener Moderne», dans Kurt Ifkovits, „Mit diesen meinen zwei Händen...", op. cit., p. 77-81.

14. Franz Servaes, «Richard Teschner - Wien», dans Deutsche Kunst und Dekoration. Wohnungskunst, Malerei, Plastik, Architektur, Gärten, künstlerische Frauenarbeiten, vol. $14, \mathrm{n}^{\circ} 12,1911$, p. 391-406.

15. Hugo Haberfeld, «Ein Damenzimmer von Richard Teschner», Dekorative Kunst, ${ }^{\circ}$ 20, 1917, p. 119-130.

16. Ibid., p. 124.

17. Voir Martina Meiderle, Richard Teschner 1879-1948. Maler, Graphiker und Kunsthandwerker. Studien anhand von ausgewählten Beispielen, Magisterarbeit, Universität Wien, 2013, p. 12. 
Franz Servaes souligne son «zèle purement artisanal» (die rein handwerkliche Tüchtigkeit), tandis que Béla Balazs compare son visage au «visage d'un brave artisan-artiste allemand du $\mathrm{xv}^{\mathrm{e}}$ siècle» (das Gesicht eines biederen deutschen Handwerkerkünstlers aus dem 15. Jahrhundert), en ajoutant plus loin que dans le romantisme d'automate qui caractérise son théâtre réside «la magie de l'artisanat pieux» (die Magie des andächtigen Handwerks ${ }^{18}$ ).

La découverte des marionnettes de Java par Richard Teschner a, en tout cas, tous les traits d'une légende. C'est pendant son voyage de noces en 1911 que Teschner aurait vu pour la première fois des marionnettes du wayang javanais ${ }^{19}$. Cette découverte aurait constitué comme une révélation. Dans son journal de voyage, il note à la date du 17 août 1911 : «Hague...chez Bocotan enfin le wayang !!!» (Haag...bei Bocotan endlich Wajang $\left.{ }^{20}\right)$. Plusieurs chercheurs ont insisté sur le fait que l'intérêt de Teschner pour l'Asie n'est pas nouveau à cette date et que, déjà à Prague, il s'était intéressé par exemple aux écrits d'Helena Blavatsky, figure importante de la Société théosophique. On a souligné aussi que le fantastique d'un autre Allemand de Bohême, Alfred Kubin, se mêlait, tout comme chez Teschner, d'inspirations asiatiques ${ }^{21}$. Quant à la particularité qui attirait particulièrement Teschner dans les marionnettes du wayang golek, à savoir le fait qu'elles sont commandées par des tiges et qu'on ne voit donc pas de fils, elle se retrouvait dans les crèches présentes dans tout l'Empire des Habsbourg depuis le XVIII ${ }^{\mathrm{e}}$ siècle et il lui aurait suffi d'aller au Volkskunde-Museum de Vienne pour en voir $^{22}$. Une note autobiographique citée par Arthur Roessler dans sa biographie de Teschner insiste sur la référence à l'« Orient» : «Découverte des Indes hollandaises / Rencontre avec des marionnettes wayang / Réveille l'ancien amour des marionnettes sous une autre forme / synthèse : romance occidentale-style oriental-technologie des tiges ${ }^{23}$.» Il est vrai

18. Béla Balazs, «Das Haus der Geheimnisse», Der Tag, 5 avril 1925, p. 3.

19. Matthew Isaac Cohen, «Richard Teschner», dans Matthew Isaac Cohen (dir.), Performing Otherness: Java and Bali on International Stages, 1905-1952, Basingstoke, Palgrave Macmillan, 2010, p. 42-47.

20. Voir Martina Meiderle, Richard Teschner 1879-1948, op. cit., p. 11.

21. Walter Zettl, «Das Phantastische bei Teschner», dans Josef Mayerhöfer (dir.), Richard Teschner (1879-1948) Puppenspieler-sezessionistischer Künstler, Vienne, Österreichische Nationalbibliothek, 1970, p. 16-19.

22. Ludwig Krafft, «Der Figurenspiegel Richard Teschners und das Puppenspiel seiner Zeit», dans Josef Mayerhöfer (dir.), Richard Teschner (1879-1948) Puppenspielersezessionistischer Künstler, op. cit., p. 10-15.

23. Arthur Roessler, Richard Teschner, Vienne, Gerlach und Wiedling, 1947, p. 153. 
aussi que Franz Servaes dénotait chez lui la présence d'une orientation «orientale», qualifiée de japonaise, poussée à l'extrême tout autant que l'autre orientation principale du style décoratif moderne, à savoir le Biedermeier, les deux se mélangeant harmonieusement chez Teschner selon lui ${ }^{24}$.

Si Teschner eut besoin d'aller chercher à Amsterdam le contact avec les marionnettes javanaises avant de faire de ce type de théâtre son activité principale, ce n'est pas seulement parce qu'il s'agissait de donner un vernis exotique à une pratique autochtone, mais de trouver une solution originale à un dilemme sans doute à la fois artistique et politicoculturel. Plusieurs éléments tendent effectivement à montrer que la synthèse que Teschner décrit dans son journal lui permit de dépasser l'opposition Prague-Vienne, le provincialisme supposé de Bohême dans une pratique qu'on pourrait qualifier de globale, autant du point de vue des formes, que de celui du contenu, marqué par l'invention d'un nouveau fantastique ${ }^{25}$.

Toujours est-il que Teschner rapporta des marionnettes javanaises de ce voyage de noces (neuf figures de théâtre d'ombres en cuir peint et quatre figures de wayang golek en bois ${ }^{26}$ ), qui constituèrent le premier noyau de son célèbre théâtre de marionnettes, mais aussi des livres liés à l'art de la marionnette et à la mythologie javanaise ${ }^{27}$. Teschner n'est pas le seul à l'époque à s'intéresser au wayang, c'est le cas également de Craig par exemple. Selon Cohen, ce dernier en a toutefois une approche plus «théorique» que Teschner ${ }^{28}$. De retour à Vienne, Teschner organise effectivement en 1912 ses premières représentations avec les marionnettes à tige rapportées d'Amsterdam sur la scène du Goldener Schrein. Il s'agit de mises en scène sans paroles de trois contes javanais ne faisant pas partie du répertoire traditionnel du wayang, mais empruntés à une compilation allemande ${ }^{29}$. Dans un premier temps, ces représentations sont privées et l'on sait que Gustav Klimt, Josef Hoffmann, Alfred Roller et Kolo Moser y assistèrent. Par la suite Teschner complète les

24. Franz Servaes, «Richard Teschner - Wien», art. cité, p. 391.

25. Veronika Schmeer, «Biographische und künstlerische Hintergründe zu Richard Teschner", dans Veronika Schmeer, Inszenierung des Unheimlichen: Prag als Topos. Buchillustrationen der deutschsprachigen Prager Moderne (1914-1925), Göttingen, V\&R Unipress, 2015, p. 58-61.

26. Voir Martina Meiderle, Richard Teschner 1879-1948, op. cit., p. 61.

27. Matthew Isaac Cohen, «Richard Teschner», art. cité, p. 42.

28. Ibid.

29. Ibid. 
marionnettes originales par des marionnettes créées par lui-même dans le même style, de manière à enrichir aussi son répertoire. Un assistant de Max Reinhardt - sachant que ce dernier assista à certaines représentations de Teschner - le contacte pendant la première guerre mondiale en vue de la réalisation d'une pièce pour Noël. La collaboration n'aura finalement pas lieu, mais Teschner réalisa seul en 1917-1918 le Weihnachtsspiel qui est une de ses créations les plus connues.

Une première exposition personnelle est consacrée à Teschner dans le cadre de l'exposition Ausstellung österreichischer Kunstgewerbe qui se tient au Museum für Kunst und Industrie entre décembre 1919 et mars 1920. Elle ne comporte pas exclusivement des marionnettes, mais c'est bien lors de cette exposition qu'eurent lieu les premières représentations publiques du théâtre de marionnettes de Teschner. Hugo von Hofmannsthal dira que dans cette exposition, c'étaient celles-ci qui l'avaient intéressé le plus, et de loin ${ }^{30}$. Le long compte rendu de l'exposition rédigé par l'architecte et écrivain Hartwig Fischel pour la revue mensuelle du Musée d'art et d'industrie, Kunst und Kunsthandwerk, est consacré pour une bonne moitié à la Sonderausstellung Richard Teschners. S'il insiste lui aussi sur le fait que l'art de Teschner prend ses racines dans l'artisanat d'art (Kunsthandwerk), l'article constitue bien un vibrant hommage à l'artiste Teschner. Il retrace minutieusement la scénographie de l'exposition avec une «entrée » où trône une Hausgöttin puis une pièce d'exposition et de représentation théâtrale dont le noyau est formé par le Goldener Schrein.

Toute une partie de l'article étudie ce que Fischel décrit comme la rencontre entre Orient et Occident dans l'art de Teschner, marquée par une "atmosphère exotico-romantique» et rappelant «quels rapports étroits ont autrefois relié l'Autriche à l'Orient ${ }^{31} »$. Au-delà des créations de Teschner, Fischel insiste sur l'affinité entre les arts décoratifs asiatiques et les arts des paysans des montagnes autrichiennes pour lesquels l'art ornemental de l'Orient (die Schmuckkunst des Orients) ne «signifie pas un monde étranger, mais un monde apparenté» (keine fremde, sondern eine verwandte Welt bedeutet) ${ }^{32}$. Pour finir, les représentations du

30. Kurt Ifkovits, «„Von meinem Hirn bis zum Maul“ oder „Vom Kopf in die Hand“. Einleitende Bemerkungen», dans Matthew Isaac Cohen (dir.), Performing Otherness, op. cit., p. 9-11, ici p. 9.

31. Hartwig Fischel, «Ausstellung Österreichischer Kunstgewerbe», Kunst und Kunsthandwerk. Monatsschrift herausgegeben vom Österreichischen Museum für Kunst und Industrie, vol. 22, no 11-12, 1919, p. 364-380, ici p. 367.

32. Ibid., p. 374. 
Goldener Schrein sont caractérisées comme une "œuvre d'art totale» (Gesamtkunstwerk) en miniature qui constitue en ces temps difficiles de l'après-guerre «un monde féérique, dans lequel nous mène la main savante, légère et fine de Teschner» (eine erlösende Märchenwelt, in welche uns die kundige, leichte und feine Hand Teschners führt) ${ }^{33}$.

À partir de 1925, les représentations eurent lieu dans l'atelier de Teschner à Gersthof et devinrent une des activités principales de l'artiste, source d'une renommée qui dépassa les frontières de l'Autriche. Le Goldener Schrein est remplacé en 1931, notamment suite aux expérimentations cinématographiques de Teschner, par une nouvelle construction scénique, le Figurenspiegel, où le marionnettiste se tient derrière un miroir convexe, voit les marionnettes et le public, mais ne peut être vu de ce dernier, une installation que Ludwig Krafft compare à un grand œil ${ }^{34}$. Teschner se fait aider à cette époque par deux assistantes, une chimiste et une tisseuse de gobelins, pour des effets spéciaux d'une grande complexité. Il est intéressant de signaler à cet égard que Isaac Matthew Cohen insiste sur la nature potentiellement cinématique du théâtre de marionnettes de Teschner ${ }^{35}$, même si on sait que ses tentatives cinématographiques échouèren ${ }^{36}$. C'est ce caractère cinématique qui le distinguerait du wayang golek, caractérisé par une tridimensionnalité qui autorise une communication directe avec le public, les marionnettes étant par ailleurs actionnées à la vue de ce dernier. Une autre différence réside dans le fait que Teschner ne voulait pas que ses marionnettes parlent et organisait leur expression autour de gestes, de musiques et d'effets de lumière. Dans le contexte de la célèbre crise du langage du tournant du siècle, partagée également par Hofmannsthal, il rejoint ce faisant le plaidoyer pour le cinéma muet de L'Homme visible (1924) et une certaine affinité, déjà notée, avec l'auteur de ce dernier, Béla Balàzs.

Comme il a été souligné, Teschner lui-même ainsi que sa réception, notamment par son biographe Arthur Roessler, ont consolidé le mythe d'une Prague "magique», mystérieuse et vieillissante, par opposition à une Vienne moderniste et ouverte sur le monde. Si le parcours de

33. Ibid., p. 371.

34. Ludwig Krafft, «Der Figurenspiegel Richard Teschners und das Puppenspiel seiner Zeit», art. cité, ici p. 13.

35. Ibid., p. 46.

36. Voir Armin Loacker, «Unbefriedigende Verhältnisse. Richard Teschners Scheitern im Medium Film », in Kurt Ifkovits, „Mit diesen meinen zwei Händen... “, op. cit., p. 258-283. 
Teschner montre à l'évidence que Vienne offrit à certains artistes germanophones de Bohême des opportunités dont ils ne disposaient pas à Prague, il faut se garder néanmoins d'en tirer des conclusions hâtives. Il peut paraitre difficile de comprendre que ce soit précisément un futur marionnettiste qui ait eu besoin de s'éloigner de Prague pour développer sa pratique, alors que Prague disposait, comme Teschner le soulignait lui-même, d'une longue tradition du théâtre de marionnettes. De plus, la Bohême devint bien au début du $\mathrm{xx}^{\mathrm{e}}$ siècle le lieu d'un renouveau et d'une modernisation importante du théâtre de marionnettes : plusieurs troupes furent créées dans différentes villes et développèrent un théâtre artistique qui partageait avec celui de Teschner ses emprunts au conte et son caractère d'œuvre d'art totale. Un certain nombre de créations en matière de théâtre de marionnettes en Bohême se montrèrent en réalité plus avant-gardistes que celles de Teschner. Les marionnettes de ce dernier, quant à elles, avec leur caractère hybride mêlant différentes mythologies et différents imaginaires visuels, si elles peuvent être lues comme le signe d'une globalisation de la marionnette, paraissent surtout avoir laissé une certaine empreinte dans bien des figures qui peuplent aujourd'hui le fantastique du cinéma hollywoodien ${ }^{37}$. C'est pourquoi il peut paraitre intéressant de les mettre en relation avec l'ouverture du livre de Stephen Greenblatt Marvelous Posessions. The Wonder of the New World (1991 University of Chicago Press), où ce dernier fait de son expérience d'un festival balinésien en 1986 et de la co-présence dans ce dernier de trois écrans (une représentation de marionnettes wayang, la projection de Death Wish II avec Charles Bronson et celle d'une comédie autour de riches yuppies à Jakarta) une scène emblématique de la mondialisation culturelle. L'opposition entre son angoisse de voir disparaître les anciennes traditions culturelles et le naturel avec lequel les villageois passent d'un écran à l'autre, le poussent à se demander qui assimile qui et à réfléchir à une hybridité fondamentale, à la circulation d'un capital mimétique et à une force reproductrice transformant des contacts culturels en formes nouvelles. Le théâtre de marionnettes de Teschner nous invite à voir sous ce face à face dramatisé une strate de relations plus anciennes qui renvoie à un autre moment de tension : entre les marionnettes tchèques, la culture métropolitaine viennoise et un Orient imaginaire teinté de théosophie. Envisager ces échanges dans la longue

37. Petra Paterno, «E.T.s Vorfahren. Theatermuseum zeigt Richards Teschners magisches Universum», Wiener Zeitung, 22 août 2013. 
durée permet de mettre en évidence une certaine interdépendance entre le proche et le lointain, le centre et la périphérie, ainsi qu'entre différents médias, au-delà de Vienne et de Prague et à différentes échelles.

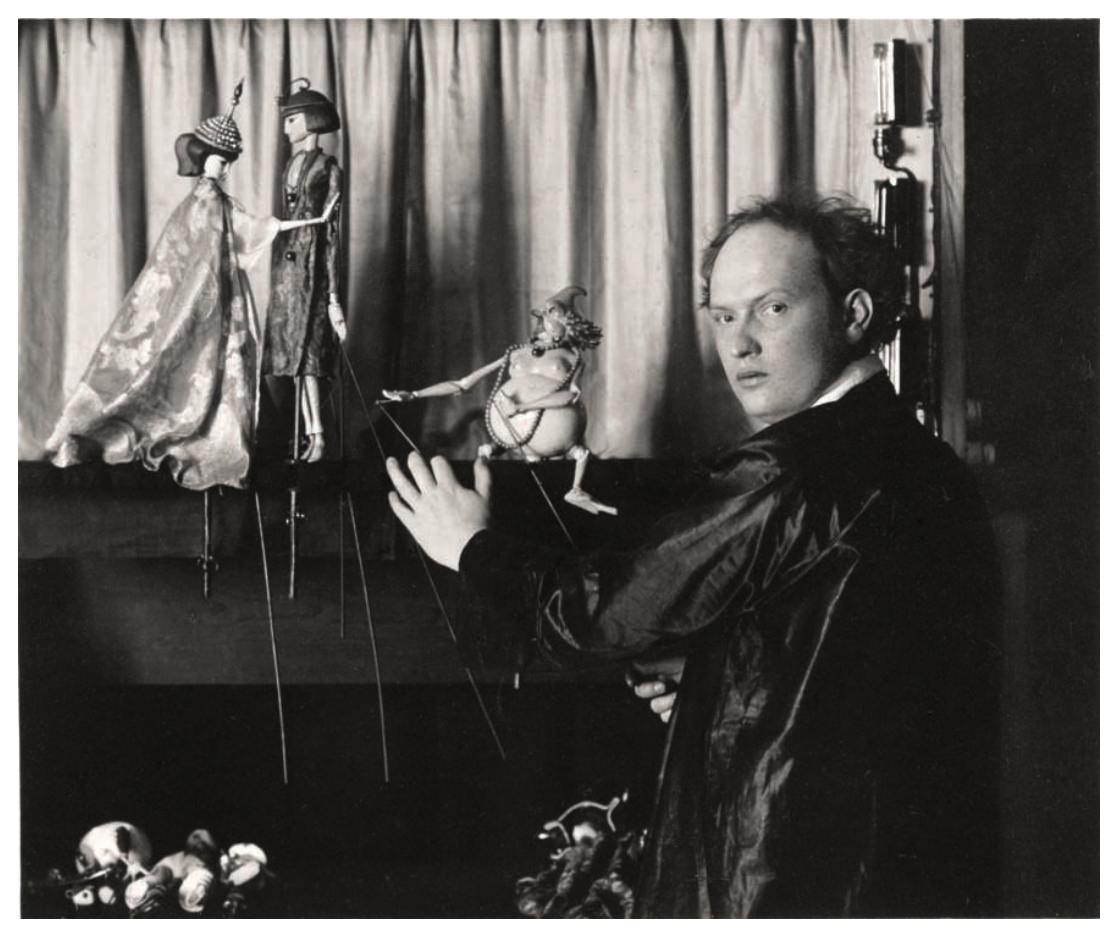

Richard Teschner mit Figuren, 1914. Photographie: Theatermuseum, Vienne.

Droits réservés. 